\title{
Influência da parição e do puerpério no leucograma de caprinos (Capra hircus) da raça Saanen, criados no Estado de São Paulo
}

Eduardo Harry BIRGEL JÚNIOR ${ }^{1}$

Rinaldo Batista VIANA ${ }^{1}$

Maria Consuelo Caribé AYRES 1

Fernando José BENESI ${ }^{1}$

Viviane GOMES'

Eduardo Harry BIRGEL ${ }^{1}$

\section{Correspondência para:}

EDUARDO HARRY BIRGEL JÚNIOR Centro de Pesquisa e Diagnóstico de

Enfermidades de Ruminantes (CPDER)

Departamento de Clínica Médica

Faculdade de Medicina Veterinária e

Zootecnia

Universidade de São Paulo

Avenida Prof. Orlando Marques de

Paiva, 87

Cidade Universitária Armando Salles de

Oliveira

05508-270 - São Paulo - SP

ehbirgel@usp.br

Recebido para publicação: 07/10/2003 Aprovado para publicação: 23/08/2005

\author{
1- Centro de Pesquisa e Diagnóstico de Enfermidades de Ruminantes \\ (CPDER) do Departamento de Clínica Médica da Faculdade de Medicina \\ Veterinária e Zootecnia da USP, São Paulo - SP
}

\section{Resumo}

Com o objetivo de avaliar a influência da parição e do puerpério no leucograma de caprinos da raça Saanen foram colhidas 360 amostras de sangue de 20 cabras, sendo os resultados apresentados em 18 grupos experimentais: 32, 16, 8, 4, 3, 2, 1 e 1/2 dias antes do parto, imediatamente após a parição, 1/2, 1, 2, 3, 4, 8, 16, 32 e 64 dias após o parto. Nas amostras de sangue colhidas em frascos contendo EDTA, foram realizadas as seguintes provas: contagem do número total de leucócitos, em câmara de Neubauer modificada, utilizando-se o líquido de Thoma como diluidor e contagem diferencial de leucócitos, efetuada em esfregaços sangüíneos, corados pelo método de Rosenfeld. A avaliação dos resultados obtidos demonstrou que o leucograma sofreu influência da parição e do puerpério, sendo que durante todo o experimento o quadro leucocitário foi predominantemente neutrofílico. Nos últimos três dias de gestação observou-se gradual aumento do número de leucócitos, em decorrência das variações observadas no número de neutrófilos. No momento do parto o leucograma era caracterizado por leucocitose devido à neutrofilia associado a linfopenia. Nas primeiras 24 horas após o parto foi observado a existência de leucocitose por neutrofilia que desapereceu nos dias subseqüentes, passando o quadro leucocitário a assemelharse entre o $2^{\circ}$ e $64^{\circ}$ dia após o parto àquele observado na fase final da gestação.

\section{Introdução}

Durante a parição, o quadro leucocitário nos caprinos é caracterizado por leucocitose devido à neutrofilia $a^{1,2,3,4}$ associado a linfopenia ${ }^{1,2,4}$ e eosinopenia ${ }^{1,2,3,4}$, estando essas alterações relacionadas ao estresse decorrente da parição e ao aumento da secreção de hormônios do córtex adrenal ${ }^{2,4}$. Com relação a influência do puerpério no quadro leucocitário foram encontradas opiniões divergentes. Parte das pesquisas relatou a ocorrência de leucocitose por neurofilia durante o puerpério ${ }^{5,6}$, enquanto a outra parte demonstrou que durante $\mathrm{O}$ puerpério ocorria a diminuição do número de leucócitos, deixando de ser observado nessa fase a leucocitose por neutrofilia que caracterizava o quadro leucocitário no momento da parição ${ }^{1,2,3,4}$. O retorno desses valores aos patamares observados no final da gestação ocorreria entre o $2^{\circ}$ e $15^{\circ}$ dia do puerpério ${ }^{1,2,3}$.

A análise da literatura que procurou avaliar a influência da parição no leucograma de caprinos demonstrou que o assunto foi objeto de estudo de somente cinco pesquisas ${ }^{1,2,3,4,7}$ e evidenciou a existência de apenas seis publicações científicas preocupadas em estudar as possíveis modificações do quadro leucocitário durante a evolução do puerpério ${ }^{1,2,3,4,5,6}$. Durante essa análise observou-se, também, que em três publicações científicas ${ }^{2,45}$ os resultados da 
contagem diferencial foram apresentados em valores absolutos, mas somente na pesquisa desenvolvida por Fortange e Schäfer ${ }^{5}$ foram apresentadas, em valores absolutos, todas as células constituintes do leucograma (neutrófilos, eosinófilos, basófilos, linfócitos e monócitos).

Os fatos expostos evidenciam que a influência da parição e do puerpério no leucograma de caprinos não foi, ainda, devidamente estudado, pois os valores absolutos dos diversos constituintes do leucograma não foram estabelecidos e existem divergências sobre a influência do puerpério no quadro leucocitário. No Brasil, o fato da influência da parição no leucograma de caprinos ter sido objeto de estudo de uma única pesquisa ${ }^{7} \mathrm{e}$ a inexistência de trabalhos científicos brasileiros que avaliem a influência do puerpério nos valores do leucograma justificam a necessidade da realização da presente pesquisas.

Assim sendo, dando com isso continuidade a linha de pesquisa desenvolvida há quatro décadas no Departamento de Clínica Médica da Faculdade de Medicina Veterinária e Zootecnia da Universidade de São Paulo, a presente pesquisa teve como objetivo estudar o quadro leucocitário de caprinos da raça Saanen, avaliando a influência da parição e do puerpério na crase sangüínea, na referida espécie.

\section{Materiais e Métodos}

Para avaliar a influência do parto e do puerpério no leucograma de caprinos, acompanhou-se, no período que compreendia o último mês de gestação até dois meses após a parição, o quadro leucocitário de 20 cabras consideradas clinicamente sadias, mantidas no Centro de Pesquisa e Diagnóstico de Enfermidades de Ruminantes (CPDER) do Departamento de Clínica Médica da Faculdade de Medicina Veterinária e Zootecnia da Universidade de São Paulo.

Nesses 20 animais foram, inicialmente, realizadas colheitas semanais de sangue, passando essas colheitas, com a aproximação do parto, a serem realizadas diariamente. Ao final da fase experimental, os resultados do leucograma dos 20 caprinos utilizados foram agrupados em 18 momentos, conforme a seguir discriminado na tabela 1 .

As amostras de sangue foram colhidas por punção da veia jugular externa, utilizando-se um sistema para colheita a vácuo constituído de agulhas $25 \times 8 \mathrm{~mm}$ para múltipla colheita, acopladas a tubos siliconizados, contendo uma solução aquosa de etileno diamino tetracetato tripotássico (EDTA-K ${ }_{3}$ ) a $15 \%$, e com vácuo suficiente para aspirar 4,5 $\mathrm{ml}$ de sangue, sendo realizadas as seguintes provas: contatem do número total de leucócitos e contagem diferencial de leucócitos.

A contagem do número total de leucócitos foi realizada em Câmara de Neubauer modificada, sendo as amostras de sangue diluídas, em pipeta hematimétrica específica, na proporção de 1:20, utilizandose como solução diluidora o líquido de Thoma de acordo com as recomendações de Birgel ${ }^{8}$. Com o sangue "in natura" foram distendidos dois esfregaços sangüíneos destinados à contagem diferencial de leucócitos. Esses esfregaços, após secarem, foram corados utilizando-se o corante de Rosenfeld, segundo técnica padronizada para os animais por Birgel ${ }^{8}$. Em cada esfregaço sangǘneo foram diferenciados 100 leucócitos classificados, de acordo com suas características morfológicas e tintoriais, em neutrófílos com núcleo em bastonete, neutrófílos com núcleo segmentado; eosinófilos, basófilos, linfócitos e monócitos.

Para calcular os valores da média aritmética e o desvio padrão, assim como avaliar a influência da gestação e do puerpério nos elementos constituintes do leucograma, utilizou-se, inicialmente, a análise de variância, sendo, a seguir, utilizado para comparação entre os pares de médias o teste de Duncan, com nível de significância igual a $5 \%$.

\section{Resultados e Discussão}

Na presente pesquisa foi demonstrada 
Tabela 1 - Constituição dos grupos experimentais para avaliação da influência da parição e do puerpério nos constituintes do hemograma de cabras da raça Saanen, criados no Estado de São Paulo. São Paulo, 2002

\begin{tabular}{|c|c|c|c|c|}
\hline & $\begin{array}{c}\text { Grupos } \\
\text { experimentais }\end{array}$ & $\begin{array}{l}\text { Número de } \\
\text { Amostras }\end{array}$ & \multicolumn{2}{|c|}{ Momento da colheita das amostras } \\
\hline \multirow{8}{*}{ 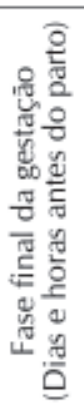 } & 1 & 20 & 32 dias ante-parto & $792 \mid-768$ horas anteparto \\
\hline & 2 & 20 & 16 dias ante-parto & $408 \mid-384$ horas ante-parto \\
\hline & 3 & 20 & 8 dias ante-parto & $216 \mid-192$ horas anteparto \\
\hline & 4 & 20 & 4 dias ante-parto & $96 \mid-72$ horas ante-parto \\
\hline & 5 & 20 & 3 dias ante-parto & $72 \mid-48$ horas ante-parto \\
\hline & 6 & 20 & 2 dias ante-parto & $48 \mid-24$ horas ante-parto \\
\hline & 7 & 20 & 1 dia ante-parto & $24 \mid-12$ horas ante-parto \\
\hline & 8 & 20 & $1 / 2$ dia ante-parto & $? 12$ horas ante -parto \\
\hline Parto & 9 & 20 & \multicolumn{2}{|c|}{ Imediatamente após o parto } \\
\hline \multirow{9}{*}{ 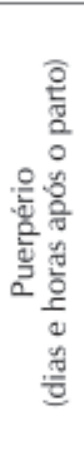 } & 10 & 20 & $1 / 2$ dia pós-parto & 12 horas pós-parto \\
\hline & 11 & 20 & 1 dia pós-parto & 24 horas pós-parto \\
\hline & 12 & 20 & 2 dias pós-parto & 48 horas pós-parto \\
\hline & 13 & 20 & 3 dias pós-parto & 72 horas pós-parto \\
\hline & 14 & 20 & 4 dias pós-parto & 96 horas pós-parto \\
\hline & 15 & 20 & 8 dias pós-parto & 192 horas pós-parto \\
\hline & 16 & 20 & 16 dias pós-parto & 384 horas pós-parto \\
\hline & 17 & 20 & 32 dias pós-parto & 768 horas pós-parto \\
\hline & 18 & 20 & 64 dias pós-parto & 1.536 horas pós-parto \\
\hline
\end{tabular}

a influência da parição no leucograma de caprinos da raça Saanen, criados no Estado de São Paulo. Os resultados obtidos estão detalhados nas tabelas 2 e 3 e na figura 1 .

Inicialmente, no período compreendido entre o $32^{\circ}$ e o $3^{\circ}$ dia antes do parto, o número de leucócitos oscilou entre $9.370 \pm 1.840$ e $9.595 \pm 2.089$ leucócitos $/ \mathrm{mm}^{3}$, sem que qualquer diferença estatística pudesse ser verificada. A seguir, nos últimos dias de gestação, foi observado o gradual aumento do número total de leucócitos. Nas amostras de sangue colhidas entre 72 e 48 horas antes do parto (3 dias antes do parto) e colhidas com menos de 12 horas antes do parto, constatou-se que os valores aumentaram, de forma significativa de $9.560 \pm 1.978$ para $12.290 \pm 2.713$ leucócitos $/ \mathrm{mm}^{3}$. A partir desse momento (últimas 12 horas antes do parto) não foram observadas diferenças estatísticas entre os valores encontrados nas amostras colhidas imediatamente após o parto (12.275 \pm 2.531 leucócitos $\left./ \mathrm{mm}^{3}\right), 12$ horas após o parto $\left(12.905 \pm 2.531\right.$ leucócitos $\left./ \mathrm{mm}^{3}\right) \mathrm{e}$ 24 horas após a parição (12.285 \pm 3.195 leucócitos / $\left.\mathrm{mm}^{3}\right)$. Exceção feita as amostras colhidas no $8^{\circ}$ dia após o parto, verificou-se que o número total de leucócitos entre o $2^{\circ}$ e o $64^{\circ}$ dias após o parto variava entre $10.350 \pm 1.895$ e 10.850 \pm 2.894 leucócitos $/ \mathrm{mm}^{3}$ e que esses valores diminuíram em relação àqueles observados no período peripartal (compreendido entre as últimas 12 horas antes e primeiras 24 horas após o parto), retornando aos patamares observados entre o $32^{\circ}$ e $3^{\circ}$ dia antes do parto.

No delineamento experimental proposto para avaliar a influência da parição e da evolução do puerpério verificou-se que as variações observadas no leucograma foram decorrentes, particularmente, às 


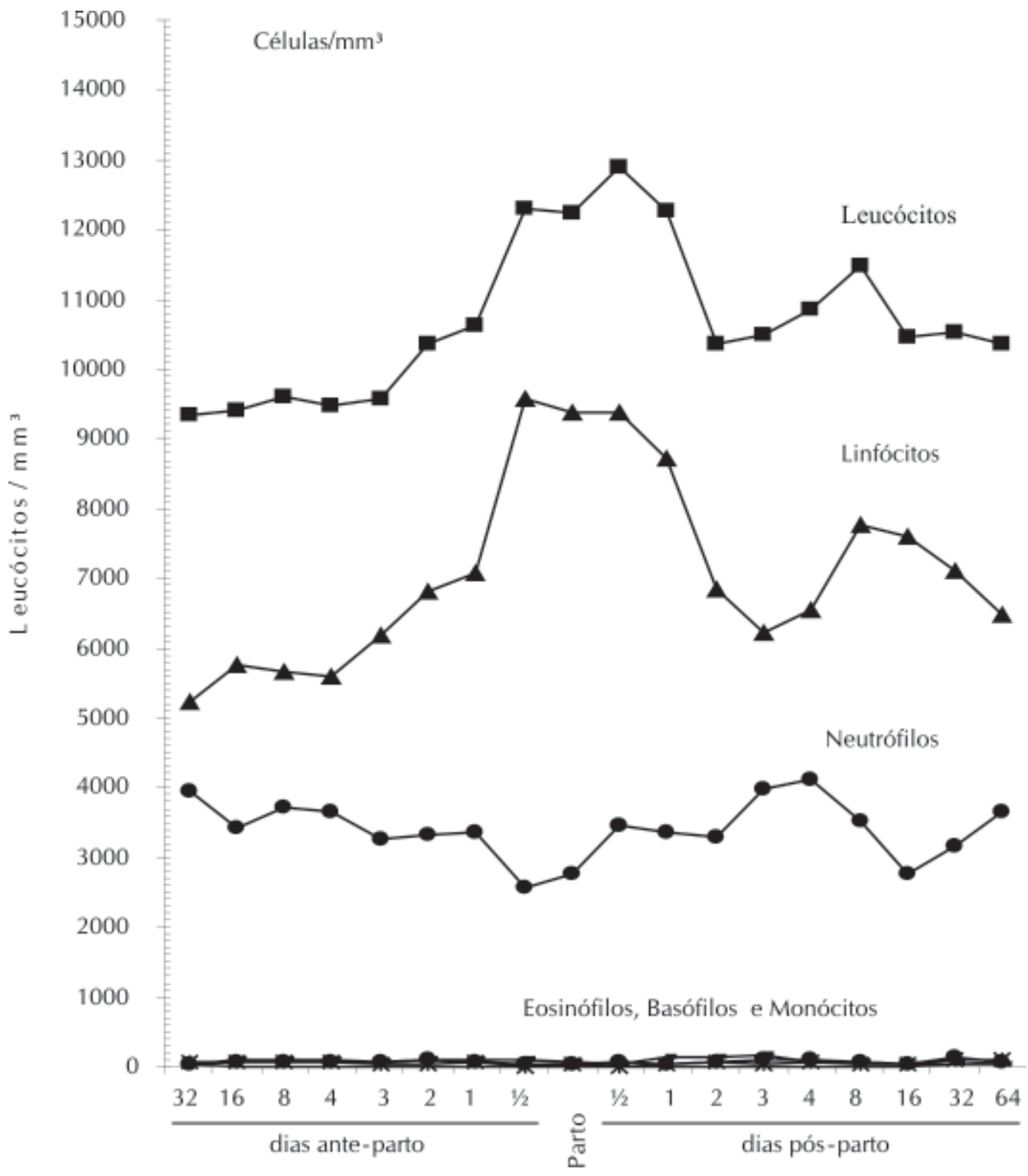

Figura 1 - Influência da parição e do puerpério no leucograma de caprinos sadios, da raça Saanen, criados no Estado de São Paulo. São Paulo-2002

alterações no número de neutrófilos. Nos três últimos dias de gestação observou-se um gradual aumento do número total de neutrófilos, sendo observado que, nas amostras de sangue colhidas com menos de 12 horas ante-parto $(9.587 \pm 2.706$ neutrófilos $/ \mathrm{mm}^{3}$ ), a diferença passa a ser estatisticamente significante. Entre 12 horas antes e 24 horas após o parto (1 dia pósparto) o valor do número total de neutrófilos permaneceu estabilizado, oscilando entre $8.722 \pm 2.980$ e $9.587 \pm 2.706$ neutrófilos / $\mathrm{mm}^{3}$. Na evolução do puerpério, no período compreendido entre o $2^{\circ}$ e o $64^{\circ}$ dia após o parto, verificou-se a diminuição do número total de neutrófilos em relação aos valores registrados no período compreendido entre as últimas 12 horas antes e as primeiras 24 horas após o parto. Nesse período do puerpério, exceção feita as amostras colhidas 
no $8^{\circ}$ e $16^{\circ}$ dia após o parto, os valores oscilaram entre $6.233 \pm 2.202$ e $7.113 \pm$ 2.669 neutrófilos $/ \mathrm{mm}^{3}$, sendo observada uma tendência dos valores retornarem aos patamares observados no final da gestação.

O número absoluto de neutrófilos com núcleo em forma de bastonete sofreu influência do puerpério. Entre 12 horas e 96 horas após o parto observaram-se os valores máximos para o número absoluto de neutrófilos bastonete, sendo esses valores encontrados nas amostras colhidas às 12 horas após o parto $(239 \pm 406$ neutrófilos bastonete $\left./ \mathrm{mm}^{3}\right), 24$ horas após o parto (268 \pm 454 neutrófilos bastonetes/ $\mathrm{mm}^{3}$ ) e às 96 horas após o parto $(246 \pm 448$ neutrófilos

Tabela 2 - Avaliação da influência da parição e do puerpério no leucograma de caprinos sadios, da raça Saanen, criados no Estado de São Paulo, segundo as características estatísticas (média, desvio padrão, e amplitude de variação). São Paulo, 2002

\begin{tabular}{|c|c|c|c|c|c|c|c|}
\hline \multicolumn{2}{|c|}{ Grupos Experimentais } & \multirow{2}{*}{$\begin{array}{c}\begin{array}{c}\text { Leucócitos } \\
\left(/ \mathrm{mm}^{3}\right)\end{array} \\
9.340 \pm 1.840^{\mathrm{2}} \\
{[5.400-12.700)}\end{array}$} & \multirow{2}{*}{$\begin{array}{c}\begin{array}{c}\text { Neutrófilos } \\
\left(/ \mathrm{mm}^{3}\right)\end{array} \\
5.237 \pm 2.087^{\mathrm{a}} \\
(2.392-9.202)\end{array}$} & \multirow{2}{*}{$\begin{array}{c}\begin{array}{c}\text { Eosinófilos } \\
\left(/ \mathrm{mm}^{3}\right)\end{array} \\
67 \pm 92^{2} \\
(0-248)\end{array}$} & \multirow{2}{*}{$\begin{array}{c}\begin{array}{c}\text { Basófilos } \\
\left(/ \mathrm{mm}^{3}\right)\end{array} \\
41 \pm 64^{2 b} \\
\langle 0-192)\end{array}$} & \multirow{2}{*}{$\begin{array}{c}\begin{array}{c}\text { Linfócitos } \\
\left(/ \mathrm{mm}^{3}\right)\end{array} \\
3.957 \pm 1.958^{\mathrm{a}} \\
(1.350-7.904)\end{array}$} & \multirow{2}{*}{$\begin{array}{c}\begin{array}{c}\text { Monócitos }(/ \\
\left.\mathrm{mm}^{3}\right)\end{array} \\
\begin{array}{c}37 \pm 80^{2} \\
(0-294)\end{array}\end{array}$} \\
\hline \multirow{8}{*}{ 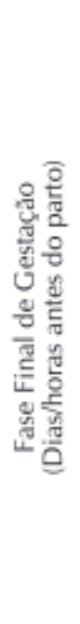 } & $\begin{array}{c}32 \text { dias } \\
\text { (792 |- } 768 \text { horas })\end{array}$ & & & & & & \\
\hline & $\begin{array}{c}16 \text { dias } \\
(408 \mid-384 \text { horas })\end{array}$ & $\begin{array}{l}9.415 \pm 1.866^{\mathrm{a}} \\
\{6.100-13.100\}\end{array}$ & $\begin{array}{l}5.767 \pm 1.925^{\mathrm{db}} \\
(1.575-9.202)\end{array}$ & $\begin{array}{l}54 \pm 76^{2} \\
(0-240)\end{array}$ & $\begin{array}{l}58 \pm 63^{\text {atc }} \\
(0-228\rangle\end{array}$ & $\begin{array}{c}3.429 \pm 1.615^{a b c} \\
(1.391-7.598)\end{array}$ & $\begin{array}{c}107 \pm 174^{2} \\
(0-590)\end{array}$ \\
\hline & $\begin{array}{c}\text { B dias } \\
\left(\begin{array}{ll|l}2 & 16 & -192 \text { horas }\end{array}\right)\end{array}$ & $\begin{array}{l}9.595 \pm 1.573^{\circ} \\
\langle 6.800-13.500\rangle\end{array}$ & $\begin{array}{l}5.669 \pm 2.168^{a b} \\
(2.886-10.935)\end{array}$ & $\begin{array}{l}64 \pm 125^{3} \\
(0-510)\end{array}$ & $\begin{array}{l}59 \pm 72^{\text {stc }} \\
(0-194)\end{array}$ & $\begin{array}{l}3.709 \pm 1.385^{3 b} \\
(1.552-6.148)\end{array}$ & $\begin{array}{l}89 \pm 139^{3} \\
(0-495)\end{array}$ \\
\hline & $\begin{array}{c}4 \text { dias } \\
(96 \mid-72 \text { horas })\end{array}$ & $\begin{array}{c}9.475 \pm 2.089^{\circ} \\
(5.800-13.400)\end{array}$ & $\begin{array}{c}5.601 \pm 2.251^{2} \\
(2.088-10.184)\end{array}$ & $\begin{array}{c}56 \pm 112^{3} \\
(0-440)\end{array}$ & $\begin{array}{l}81 \pm 81^{\text {abc }} \\
(0-246)\end{array}$ & $\begin{array}{l}3.644 \pm 1.119^{\mathrm{db}} \\
(1.896-6.035)\end{array}$ & $\begin{array}{l}93 \pm 115^{3} \\
(0-436)\end{array}$ \\
\hline & $\begin{array}{c}3 \text { dias } \\
(72 \mid-48 \text { horas })\end{array}$ & $\begin{array}{c}9.560 \pm 1.978^{a} \\
{[6.500-14.800)}\end{array}$ & $\begin{array}{l}6.186 \pm 2.365^{\mathrm{abc}} \\
(2.232-11.692)\end{array}$ & $\begin{array}{l}22 \pm 50^{2} \\
(0-182)\end{array}$ & $\begin{array}{l}55 \pm 96^{a t x} \\
(0-288)\end{array}$ & $\begin{array}{c}3.243 \pm 1.057^{\text {abc }} \\
(1.560-5.760)\end{array}$ & $\begin{array}{l}63 \pm 110^{4} \\
(0-436)\end{array}$ \\
\hline & $\begin{array}{c}2 \text { dias } \\
(48 \mid-24 \text { horas })\end{array}$ & $\begin{array}{c}10.350 \pm 2.777^{a b} \\
\{6.900-17.000\}\end{array}$ & $\begin{array}{l}6.800 \pm 2.913^{\mathrm{abx}} \\
(2.400-14.580)\end{array}$ & $\begin{array}{l}30 \pm 77^{a} \\
(0-327\rangle\end{array}$ & $\begin{array}{c}89 \pm 109^{d x c} \\
(0-340)\end{array}$ & $\begin{array}{c}3.321 \pm 1.292^{\mathrm{abc}} \\
(1.296-5.780)\end{array}$ & $\begin{array}{c}111 \pm 110^{2} \\
(0-324)\end{array}$ \\
\hline & $\begin{array}{c}1 \text { dia } \\
(24 \mid-12 \text { horas })\end{array}$ & $\begin{array}{c}10.625 \pm 2.427^{i b c} \\
(6.700-14.700)\end{array}$ & $\begin{array}{l}7.067 \pm 2.677^{\text {abod }} \\
(3.192-12.495)\end{array}$ & $\begin{array}{l}52 \pm 77^{a} \\
\langle 0-286)\end{array}$ & $\begin{array}{l}68 \pm 84^{\text {the }} \\
(0-268)\end{array}$ & $\begin{array}{c}3.350 \pm 1.313^{2 b c} \\
(1.696-6.148)\end{array}$ & $\begin{array}{l}89 \pm 117^{2} \\
(0-472)\end{array}$ \\
\hline & $\begin{array}{c}1 / 2 \text { dia } \\
\langle<12 \text { horas })\end{array}$ & $\begin{array}{l}12290 \pm 2.713^{\text {d }} \\
\{6.100-16.700\}\end{array}$ & $\begin{array}{c}9.587 \pm 2.706^{e} \\
(4.270-14.529)\end{array}$ & $\begin{array}{l}11 \pm 36^{\mathrm{a}} \\
(0-129)\end{array}$ & $\begin{array}{l}26 \pm 56^{2} \\
(0-194)\end{array}$ & $\begin{array}{l}2.557 \pm 786^{c} \\
(873-4.256)\end{array}$ & $\begin{array}{l}109 \pm 265^{2} \\
(0-1.176)\end{array}$ \\
\hline & $\begin{array}{c}12.275 \pm 2.531^{c d} \\
(8.600-16.900)\end{array}$ & $\begin{array}{c}9.377 \pm 2.980^{e f} \\
(2.912-14.760)\end{array}$ & $\begin{array}{l}18 \pm 44^{\mathrm{a}} \\
(0-142)\end{array}$ & $\begin{array}{l}26 \pm 55^{2} \\
(0-172)\end{array}$ & $\begin{array}{l}2.774 \pm 1.449^{b c} \\
(1.640-8.288)\end{array}$ & $\begin{array}{l}81 \pm 141^{2} \\
(0-507)\end{array}$ \\
\hline \multirow{9}{*}{ 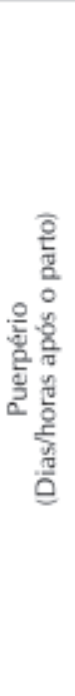 } & $\begin{array}{c}1 / 2 \text { dia } \\
\text { (12 horas) }\end{array}$ & $\begin{array}{l}12.905 \pm 2.372^{d} \\
(8.900-19.000)\end{array}$ & $\begin{array}{c}9.365 \pm 2.796^{\circ} \\
(3.738-14.440)\end{array}$ & $\begin{array}{l}5 \pm 24^{2} \\
(0-109)\end{array}$ & $\begin{array}{c}56 \pm 120^{3 b c} \\
(0-445)\end{array}$ & $\begin{array}{l}3.452 \pm 1.111^{a b c} \\
(1.781-5.715)\end{array}$ & $\begin{array}{l}27 \pm 69^{2} \\
(0-254)\end{array}$ \\
\hline & $\begin{array}{c}1 \text { dia } \\
\text { (24 horas) }\end{array}$ & $\begin{array}{c}12.285 \pm 3.195{ }^{d d} \\
(7.200-20.900)\end{array}$ & $\begin{array}{l}8.722 \pm 3.249^{\mathrm{def}} \\
(3.528-16.093)\end{array}$ & $\begin{array}{l}38 \pm 64^{2} \\
(0-208)\end{array}$ & $\begin{array}{c}48 \pm 64^{i b} \\
(0-172)\end{array}$ & $\begin{array}{c}3.361 \pm 1.380^{a b c} \\
(847-6.477)\end{array}$ & $\begin{array}{c}117 \pm 179^{2} \\
(0-627)\end{array}$ \\
\hline & $\begin{array}{c}2 \text { dias } \\
\text { (48 horas) }\end{array}$ & $\begin{array}{c}10.355 \pm 2.180^{a b} \\
(6.400-15.200)\end{array}$ & $\begin{array}{l}6.838 \pm 2.365^{3 b c} \\
(3.136-13.376)\end{array}$ & $\begin{array}{l}61 \pm 92^{2} \\
(0-324)\end{array}$ & $\begin{array}{l}56 \pm 80^{\text {atc }} \\
(0-244)\end{array}$ & $\begin{array}{c}3.276 \pm 1.325^{2 b c} \\
(1.456-6.726)\end{array}$ & $\begin{array}{c}124 \pm 189^{\circ} \\
(0-816)\end{array}$ \\
\hline & $\begin{array}{c}3 \text { dias } \\
\text { (72 horas) }\end{array}$ & $\begin{array}{c}10.485 \pm 2.213^{\text {skc }} \\
\{7.200-15.100\}\end{array}$ & $\begin{array}{l}6.233 \pm 2.202^{3 b c} \\
(3.458-10.857)\end{array}$ & $\begin{array}{l}35 \pm 50^{\circ} \\
(0-112)\end{array}$ & $\begin{array}{l}96 \pm 101^{b c} \\
(0-302)\end{array}$ & $\begin{array}{l}3.971 \pm 1.463^{\circ} \\
(1.980-7.668)\end{array}$ & $\begin{array}{c}149 \pm 174^{2} \\
(0-568)\end{array}$ \\
\hline & $\begin{array}{c}4 \text { dias } \\
\text { (96 horas) }\end{array}$ & $\begin{array}{c}10.850 \pm 2.894^{\dot{x}} \\
(6 . .900-19.700)\end{array}$ & $\begin{array}{l}6.534 \pm 2.973^{\text {abc }} \\
(3.078-13.987)\end{array}$ & $\begin{array}{l}52 \pm 117^{4} \\
(0-475)\end{array}$ & $\begin{array}{c}84 \pm 102^{d x} \\
(0-332)\end{array}$ & $\begin{array}{l}4.104 \pm 1.435^{a} \\
(2.484-8.322)\end{array}$ & $\begin{array}{l}76 \pm 124^{a} \\
(0-483)\end{array}$ \\
\hline & $\begin{array}{c}8 \text { dias } \\
\text { (192 horas) }\end{array}$ & $\begin{array}{c}11.480 \pm 4.059^{\text {bred }} \\
(6.600-19.000)\end{array}$ & $\begin{array}{l}7.760 \pm 3.434^{\mathrm{cdf}} \\
(4.080-14.118)\end{array}$ & $\begin{array}{l}36 \pm 73^{a} \\
(0-255)\end{array}$ & $\begin{array}{c}71 \pm 102^{d x c} \\
(0-320)\end{array}$ & $\begin{array}{c}3.532 \pm 1.095^{\mathrm{abc}} \\
(1.701-5.700)\end{array}$ & $\begin{array}{l}82 \pm 148^{4} \\
(0-549)\end{array}$ \\
\hline & $\begin{array}{c}16 \text { dias } \\
\text { (384 horas) }\end{array}$ & $\begin{array}{l}10.450 \pm 2.388^{i b} \\
(7.100-17.400)\end{array}$ & $\begin{array}{l}7.588 \pm 2.611^{\text {bodf }} \\
(3.510-14.790)\end{array}$ & $\begin{array}{l}38 \pm 64^{2} \\
\langle 0-200\rangle\end{array}$ & $\begin{array}{l}22 \pm 58^{2} \\
(0-226)\end{array}$ & $\begin{array}{c}2.774 \pm 932^{b c} \\
(990-4.600)\end{array}$ & $\begin{array}{l}29 \pm 85^{2} \\
(0-360)\end{array}$ \\
\hline & $\begin{array}{c}32 \text { dias } \\
\text { (768 horas) }\end{array}$ & $\begin{array}{c}10.535 \pm 2.544^{\text {stc }} \\
(6.200-18.600)\end{array}$ & $\begin{array}{c}7.113 \pm 2.669^{\text {abod }} \\
(3.315-15.066)\end{array}$ & $\begin{array}{l}88 \pm 137^{2} \\
(0-588)\end{array}$ & $\begin{array}{c}120 \pm 135^{c} \\
(0-492)\end{array}$ & $\begin{array}{c}3.167 \pm 1.497^{2 b c} \\
(980-7.011)\end{array}$ & $\begin{array}{l}47 \pm 95^{3} \\
(0-336)\end{array}$ \\
\hline & $\begin{array}{c}64 \text { dias } \\
\text { (1.536 horas) }\end{array}$ & $\begin{array}{c}10.350 \pm 1.895^{\mathrm{bN}} \\
(7.700-14.100)\end{array}$ & $\begin{array}{l}6.492 \pm 1.794^{\text {stc }} \\
(4.094-10.575)\end{array}$ & $\begin{array}{l}91 \pm 141^{3} \\
\langle 0-585\rangle\end{array}$ & $\begin{array}{l}63 \pm 78^{\text {the }} \\
(0-240)\end{array}$ & $\begin{array}{l}3.667 \pm 1.240^{3 b} \\
(2.436-7.398)\end{array}$ & $\begin{array}{l}37 \pm 66^{2} \\
(0-249)\end{array}$ \\
\hline
\end{tabular}

a,b,c,d,e,f- letras sobrescritas não coincidentes denotam diferença estatística significante. (P£ 0,05) - Teste de Duncan 
Tabela 3 - Avaliação da influência da parição e do puerpério no número de neutrófilos de caprinos sadios, da raça Saanen, criados no Estado de São Paulo, segundo as características estatísticas (média, desvio padrão, e amplitude de variação). São Paulo, 2002

\begin{tabular}{|c|c|c|c|c|}
\hline \multicolumn{2}{|c|}{ Grupos Experimentais } & \multirow{2}{*}{$\begin{array}{c}\begin{array}{c}\text { Neutrófilos } \\
\text { bastonetes } \\
\left(/ \mathrm{mm}^{3} \mathrm{l}\right)\end{array} \\
\begin{array}{c}17 \pm 42^{\mathrm{ab}} \\
(0-124)\end{array}\end{array}$} & \multirow{2}{*}{$\begin{array}{c}\text { Neutrófilos segmentados } \\
\left(/ \mathrm{mm}^{3} \mid\right)\end{array}$} & \multirow{2}{*}{$\begin{array}{c}\text { Total de Neutrófilos } \\
\qquad\left(/ \mathrm{mm}^{3} \mid\right) \\
\begin{array}{l}5.237 \pm 2.087^{\mathrm{a}} \\
(2.392-9.202)\end{array}\end{array}$} \\
\hline \multirow{14}{*}{ 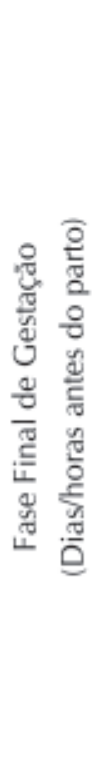 } & $\begin{array}{c}32 \text { dias } \\
\text { (792|- } 768 \text { horas) }\end{array}$ & & & \\
\hline & $\begin{array}{c}16 \text { dias } \\
\text { (408|- } 384 \text { horas) }\end{array}$ & $\begin{array}{l}11 \pm 51^{\mathrm{ab}} \\
(0-228)\end{array}$ & $\begin{array}{l}5.756 \pm 1.928^{\mathrm{ab}} \\
(1.575-9.202)\end{array}$ & $\begin{array}{l}5.767 \pm 1.925^{\text {b }} \\
(1.575-9.202)\end{array}$ \\
\hline & 8 dias & $19 \pm 50^{a b}$ & $5.650 \pm 2.163^{a b}$ & $5.669 \pm 2.168^{a b}$ \\
\hline & (216 |-192 horas) & $(0-192)$ & $(2.886-10.935)$ & $(2.886-10.935)$ \\
\hline & 4 dias & $15 \pm 49^{a b}$ & $5.586 \pm 2.255^{\mathrm{a}}$ & $5.601 \pm 2.251^{\mathrm{a}}$ \\
\hline & (96 |-72 horas) & $(0-194)$ & $(1.972-10.184)$ & $(2.088-10.184)$ \\
\hline & 3 dias & $38 \pm 85^{a b}$ & $6.149 \pm 2.383^{a b c}$ & $6.186 \pm 2.365^{a b c}$ \\
\hline & (72 |- 48 horas) & $(0-304)$ & $(2.088-11.692)$ & $(2.232-11.692)$ \\
\hline & 2 dias & $5 \pm 22^{a}$ & $6.795 \pm 2.914^{\text {abcd }}$ & $6.800 \pm 2.913^{\mathrm{abc}}$ \\
\hline & (48 |- 24 horas) & $(0-98)$ & $(2.400-14.580)$ & $(2.400-14.580)$ \\
\hline & $1 \mathrm{dia}$ & $5 \pm 22^{a}$ & $7.062 \pm 2.678^{\text {abcd }}$ & $7.067 \pm 2.677^{\mathrm{abcd}}$ \\
\hline & (24 | - 12 horas) & $(0-97)$ & $(3.192-12.495)$ & $(3.192-12.495)$ \\
\hline & $1 / 2$ dia & $48 \pm 69^{\mathrm{ab}}$ & $9.539 \pm 2.706^{e}$ & $9.587 \pm 2.706^{e}$ \\
\hline & ( $<12$ horas) & $(0-184)$ & $(4.270-14.529)$ & $(4.270-14.529)$ \\
\hline \multicolumn{2}{|r|}{ Parto } & $\begin{array}{c}127 \pm 207^{\text {abc }} \\
(0-846)\end{array}$ & $\begin{array}{c}9.249 \pm 2.984^{\text {ef }} \\
(2.912-14.596)\end{array}$ & $\begin{array}{c}9.377 \pm 2.980^{\text {ef }} \\
(2.912-14.760)\end{array}$ \\
\hline \multirow{18}{*}{ 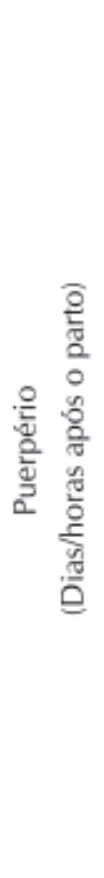 } & $1 / 2 \mathrm{dia}$ & $239 \pm 406^{c}$ & $9.126 \pm 2.715^{\text {ef }}$ & $9.365 \pm 2.796^{\text {ef }}$ \\
\hline & (12 horas) & $(0-1.395)$ & $(3.738-14.440)$ & $(3.738-14.440)$ \\
\hline & $1 \mathrm{dia}$ & $268 \pm 454^{c}$ & $8.454 \pm 3.233^{\mathrm{def}}$ & $8.722 \pm 3.249^{\text {def }}$ \\
\hline & (24 horas) & $(0-1.300)$ & $(3.312-16.093)$ & $(3.528-16.093)$ \\
\hline & 2 dias & $134 \pm 213^{\mathrm{abc}}$ & $6.704 \pm 2.365^{\text {abcd }}$ & $6.838 \pm 2.365^{a b c}$ \\
\hline & (48 horas) & $(0-756)$ & $(3.136-13.224)$ & $(3.136-13.376)$ \\
\hline & 3 dias & $166 \pm 291^{b c}$ & $6.067 \pm 2.104^{\mathrm{abc}}$ & $6.233 \pm 2.202^{\text {ahc }}$ \\
\hline & (72 horas) & $(0-906)$ & $(3.458-10.716)$ & $(3.458-10.857)$ \\
\hline & 4 dias & $246 \pm 448^{c}$ & $6.288 \pm 2.657^{\mathrm{abc}}$ & $6.534 \pm 2.973^{a b c}$ \\
\hline & (96 horas) & $(0-1.970)$ & $(2.964-12.324)$ & $(3.078-13.987)$ \\
\hline & 8 dias & $58 \pm 116^{a b}$ & $7.702 \pm 3.389^{\mathrm{cdf}}$ & $7.760 \pm 3.434^{\text {cdf }}$ \\
\hline & (192 horas) & $(0-356)$ & $(3.995-13.937)$ & $(4.080-14.118)$ \\
\hline & 16 dias & $27 \pm 81^{a b}$ & $7.561 \pm 2.559^{\text {bodf }}$ & $7.588 \pm 2.611^{\text {bodf }}$ \\
\hline & (384 horas) & $(0-348)$ & $(3.510-14.442)$ & $(3.510-14.790)$ \\
\hline & 32 dias & $18 \pm 61^{a b}$ & $7.095 \pm 2.662^{\text {abcd }}$ & $7.113 \pm 2.669^{\text {abcd }}$ \\
\hline & (768 horas) & $(0-254)$ & $(3.315-15.066)$ & $(3.315-15.066)$ \\
\hline & 64 dias & $17 \pm 35^{\mathrm{ab}}$ & $6.475 \pm 1.806^{\mathrm{abc}}$ & $6.492 \pm 1.794^{a b c}$ \\
\hline & (1.536 horas) & $(0-97)$ & $(4.094-10.575)$ & $(4.094-10.575)$ \\
\hline
\end{tabular}

a,b,c,d,e,f- letras sobrescritas não coincidentes denotam diferença estatística significante. (P£ 0,05) - Teste de Duncan 
bastonete $/ \mathrm{mm}^{3}$ ), significativamente maiores do que os observados nas cabras no final da gestação.

Verificou-se que as variações observadas para o número total de neutrófilos foram decorrentes, particularmente, do comportamento do número de neutrófilos com núcleo segmentado, sendo a curva para as duas variáveis semelhantes. Nos últimos três dias de gestação ocorreu gradual aumento do número absoluto de neutófilos segmentado, sendo verificado que, nas amostras de sangue colhidas com menos de 12 horas ante-parto $(9.539 \pm 2.706$ neutrófilos segmentado / $\mathrm{mm}^{3}$ ), as diferenças passam a ser estatisticamente significantes. A seguir, observou-se que entre 12 horas antes e 24 horas após o parto, o número absoluto de neutrófilos segmentado permaneceu estabilizado, com os valores oscilando entre $8.454 \pm 3.233$ e $9.539 \pm$ 2.706 neutrófilos segmentado $/ \mathrm{mm}^{3}$. Na evolução do puerpério, entre o $2^{\circ}$ e $064^{\circ}$ dia após o parto, verificou-se uma tendência dos valores serem menores do que aqueles registrados no período compreendido entre as últimas 12 horas antes do parto e as primeiras 24 horas de puerpério, pois nas amostras de sangue colhidas no $3^{\circ}, 4^{\circ}$ e $64^{\circ}$ dia após o parto, os números absolutos de neutrófilos com núcleo segmentado, respectivamente, iguais a $6.725 \pm 2.137,6.836$ \pm 3.118 e $7.441 \pm 1774$ neutrófilos segmentado $/ \mathrm{mm}^{3}$, foram estatisticamente menores do que aqueles encontrados nas amostras colhidas nas últimas 12 horas de gestação. $\mathrm{Na}$ evolução do puerpério, no período compreendido entre o $2^{\circ}$ e o $64^{\circ}$ dia após o parto, verificou-se a diminuição do número total de neutrófilos em relação aos valores registrados no período compreendido entre as últimas 12 horas antes e as primeiras 24 horas após o parto. Nesse período do puerpério, exceção feita as amostras colhidas no $8^{\circ}$ e $16^{\circ}$ dia após o parto, os valores oscilaram entre $6.067 \pm$ 2.104 e $7.095 \pm 2.662$ neutrófilos segmentado/ $\mathrm{mm}^{3}$, sendo observada uma diferença estatisticamente significante e uma tendência dos valores retornarem aos patamares observados entre o $32^{\circ}$ e $3^{\circ}$ dia antes do parto.

O número absoluto de eosinófilos não sofreu influência da parição, pois os valores obtidos nos últimos 32 dias de gestação e nos primeiros 64 dias após o parto oscilaram entre $5 \pm 24$ e $91 \pm 88$ eosinófilos/ $\mathrm{mm}^{3}$ sem que qualquer diferença estatística significante fosse observada.

Os valores obtidos para o número absoluto de basófilos oscilaram durante a fase final de gestação e durante a evolução do puerpério de uma forma aleatória entre $22 \pm 58$ e $120 \pm 135$ basófilos $/ \mathrm{mm}^{3}$. Apesar dos valores obtidos nas amostras colhidas 3 dias após a parição $(96 \pm 101$ basófilos $/ \mathrm{mm}^{3}$ e 32 dias após a parição $\left(120 \pm 135\right.$ basófilos $\left./ \mathrm{mm}^{3}\right)$ terem sido estatisticamente diferentes daqueles obtidos nos demais grupos experimentais não foi possível afirmar a natureza das variações observadas.

A avaliação dos resultados para o número de linfócitos evidenciou que essa variável sofreu influência da parição, sendo o quadro leucocitário, durante o experimento proposto para avaliar a influência da parição e da evolução do puerpério, predominantemente neutrofílico. Os resultados demonstraram a existência de diferenças estatisticamente significantes, pois o número de linfócitos observado nas amostras colhidas 12 horas antes do parto $\left(2.557 \pm 786\right.$ linfócitos $\left./ \mathrm{mm}^{3}\right)$ e colhidas imediatamente após o parto $(2.774 \pm 1.449$ linfócitos $/ \mathrm{mm}^{3}$ ) foram menores do que os valores encontrados nas amostras colhidas 32 dias (3.957 \pm 1.958 linfócitos $\left./ \mathrm{mm}^{3}\right), 8$ dias $\left(3.709 \pm 1.385\right.$ linfócitos $\left./ \mathrm{mm}^{3}\right)$ e 4 dias antes do parto (3.644 \pm 1.119 linfócitos / $\left.\mathrm{mm}^{3}\right)$. Durante a evolução do puerpério observou-se que os valores obtidos nas amostras colhidas 16 dias após a parição $\left(2.774 \pm 932\right.$ linfócitos $\left./ \mathrm{mm}^{3}\right)$ foram estatisticamente menores daqueles obtidos nas amostras de sangue colhidas no $3^{\circ}$ e $4^{\circ}$ dia após o parto, respectivamente, iguais a 
$3.971 \pm 1.463$ e $4.104 \pm 1.435$ linfócitos / $\mathrm{mm}^{3}$, entretanto, não foi possível afirmar a natureza das variações observadas, pois não houve nesse período tendência de aumento ou diminuição desses valores.

O número absoluto de monócitos não sofreu influência da parição, pois os valores obtidos nos últimos 32 dias de gestação e nos primeiros 64 dias após o parto oscilaram entre $27 \pm 69$ e $149 \pm 174$ monócitos $/ \mathrm{mm}^{3}$ sem que qualquer diferença estatística significante fosse observada.

A constatação da parição como responsável por uma leucocitose corrobora os resultados descritos por diversos autores que avaliaram a influência da parição no leucograma de caprinos ${ }^{1,2,3,4}$. Independente da espécie estudada, a análise da literatura mundial sobre a influência da parição no leucograma demonstrou uma concordância relativa aos efeitos do parto no número de leucócitos. Dessa forma, os resultados obtidos na presente pesquisa, bem como aqueles compulsados na literatura estão em discordância com a opinião de Marques Junior, Silva e Batista ${ }^{7}$, pois os referidos autores não mencionaram qualquer alteração no número de leucócitos durante a parição.

Segundo o delineamento proposto para avaliar a influência da parição no leucograma de caprinos sadios da raça Saanen, criados no Estado de São Paulo, demonstrou-se que as modificações observadas no leucograma nos últimos três dias de gestação foram decorrentes às variações ocorridas, principalmente, no número absoluto de neutrófilos segmentado. A constatação da existência de leucocitose por neutrofilia no momento da parição nas cabras está em concordância com as observações referidas na literatura mundial ${ }^{1,2,3,4}$, devendo essas alterações estarem relacionadas ao estresse decorrente da parição e associadas ao aumento da secreção de hormônios do córtex adrenal ${ }^{2,4}$.

Afora essa modificações foi demonstrado, na presente pequisa, a ocorrência de linfopenia absoluta associado ao parto, confirmando as observações de autores que relataram a existência de linfopenia relativa associada ao parto de caprinos $^{1,2,4}$. Entretanto, não foi demonstrada a eosinopenia descrita por vários autores para a espécie caprina ${ }^{1,2,3,4}$.

$\mathrm{Na}$ presente pesquisa o número absoluto de basófilos e o número absoluto de monócitos não sofreram influência da parição nos seus valores. Dessa forma discordou-se dos autores ${ }^{4}$ que relataram, em caprinos, um aumento do número relativo de basófilos durante o parto.

Evidenciou-se pelos resultados obtidos na presente pesquisa, que o quadro leucocitário sofreu, na fase inicial do puerpério, intensas modificações com a diminuição do número de leucócitos, deixando de ser observado o quadro de leucocitose devido à neutrofilia que caracteriza o quadro leucocitário no momento da parição. Diversos autores que trabalharam com hematologia de caprinos relataram em seus estudos um fenômeno semelhante ${ }^{1,2,3,4}$. Divergências foram verificadas em relação ao momento do puerpério, no qual os valores do leucograma retornariam aos patamares observados no final da gestação. Na presente pesquisa assim como na pesquisa desenvolvida por Roy, Sahni e Datta ${ }^{2}$ esse retorno ocorreu 48 horas (dois dias) após a parição, já pelos resultados apresentados por Azab e Abdel-Maksoud ${ }^{1}$ esse fato ocorreu uma semana após o parto, enquanto para Verma, Pandit e $\mathrm{Nema}^{3}$ isso ocorreu por volta do $15^{\circ}$ dia de puerpério.

Em contrapartida, as afirmações ${ }^{5,6}$ que durante o puerpério ocorria um aumento do número de leucócitos devido à neutrofilia, estão em discordância com os resultados obtidos na presente pesquisa, pois entre 15 e 20 dias após o parto o quadro leucocitário, no delineamento experimental desenvolvido para avaliar a influência do puerpério, foi claramente diferente do quadro leucocitário descrito por esses autores ${ }^{5,6}$.

\section{Conclusões}

A avaliação dos resultados obtidos 
demonstrou que o leucograma sofreu influência da parição e do puerpério, sendo que durante todo o experimento o quadro leucocitário foi predominantemente neutrofílico. Nos últimos três dias de gestação observou-se gradual aumento do número de leucócitos, em decorrência das variações observadas no número de neutrófilos. No momento do parto, o leucograma era caracterizado por leucocitose devido à neutrofilia associado a linfopenia. Nas primeiras
24 horas após o parto foi observado a existência de leucocitose por neutrofilia que desapereceu nos dias subseqüentes, passando o quadro leucocitário a assemelhar-se entre o $2^{\circ}$ e $64^{\circ}$ dia após o parto àquele observado na fase final da gestação.

\section{Agradecimentos}

Pesquisa financiada pela Fundação de Amparo à Pesquisa do Estado de São Paulo - FAPESP - Processo no 99/06814-0.

\title{
Influence of parturition and puerperium in the leucogram of Saanen goats (Capra hircus), raised in the State of São Paulo - Brazil
}

\begin{abstract}
With the intention of evaluating the influence of parturition and puerperium on the leukogram of Saanen goats (Capra hircus) 360 blood samples were collected from 20 goats and allotted into 18 experimental groups as described: $32,16,8,4,3,2,1 \mathrm{e}^{1 / 2}$ days before parturition, immediately after parturition, $1 / 2,1,2,3,4,8,16,32$ e 64 days after parturition. The blood samples were collected with EDTA and submitted to the following tests: total leukocyte counts, at the modified Neubauer hemocytometer, using Thomas liquid as a dilute and differential leukocyte count, made up with blood smears stained with Rosenfeld method. The evaluation of the leukogram showed significant variation that could be attributed to parturition and the puerperium. The leucograma became mostly neutrophilic at the end of pregnancy and puerperium. On the last three days of pregnancy, a gradual raise on the number of leukocytes was observed due to same changes on the total number of neutrophils. At the moment of parturition the leukogram was characterized by a leukocytosis due to neutrhophilia and lymphopenia. On the first 24 hours after parturition, it was still possible to observe this leukocytosis due to neutrophilia that disappeared on the subsequent days making the leukogram look similar to taht obseved at the late pregnancy until the end of puerperium.
\end{abstract}

Key-words: Leukogram.

\section{Referências}

1 AZAB, E. M.; ABDEL-MAKSOUD, H. A. Changes in some hematological and biochemical parameters during prepartum and postpartum periods in female Baladi goats. Small Ruminant Research, v. 34, n. 1, p. 77-85, 1999.

2 ROY, A.; SAHNI, K. L.; DATTA, I. C. Studies on certain aspects of sheep and goat husbandry. VII variations in blood corpuscles of sheep and goat during different seasons, pregnancy, parturition and postparturition period. Indian Journal Veterinary Science, v. 35, n. 1, p. 24-32, 1965.

3 VERMA, A. K.; PANDIT, R. K.; NEMA, S. P. Certain observations on parturition process in local goats. Indian Veterinary Journal, v. 67, n. 4, p. 376-377, 1990.

4 VIHAN, V. A.; RAI, P. Certain hematological and biochemical attributes during pregnancy, parturition and pos-parturition periods in sheep and goats. Indian Journal of Animal Sciences, v. 57, n. 11, p. 12001204, 1987.

5 FORTAGNE, M.; SCHÄFER, M. Hämatologische parameter der Probstheidaer kleinziege in abhängigkeit von gravidität und laktation. Archiv fur Experimentelle Veterinarmedizin. v, 43, p. 223-230, 1989. 
6 MBASSA, G. K.; POULSEN, J. S. D. Influence of pregnancy, lactation and environment on haematology profiles in Danish Landrace dairy goats (Capra hircus) of different parity. Comparative Biochemistry Physiology - B, v. 100, n. 2, p. 403-412, 1991.

7 MARQUES JÚNIOR, A. P.; SILVA, T. M. F.; BATISTA, R. A. Hemograma de cabras leiteiras nos períodos pré e pós-parto, mantidas em confinamento. Arquivo Brasileiro de Medicina Veterinária e Zootecnia, v. 42, n. 3, p. 187-195, 1990.

8 BIRGEL, E. H. Hematologia clínica veterinária. In: BIRGEL, E. H.; BENESI, F. J. Patologia clínica veterinária. São Paulo: Sociedade Paulista de Medicina Veterinária, 1982. p. 2-34.

9 BERQUÓ, E. S.; SOUZA, J. M. P.; GOTLIEB, S. L. D. Bioestatística. São Paulo: Pedagógica e Universitária, 1980. $325 \mathrm{p}$. 